\title{
MOLYBDENUM COFACTOR DEFICIENCY: DIETARY THERAPY
}

Moderate short-term clinical improvement was obtained with dietary methionine restriction and cysteine supplementation in a 5-month-old-girl with molybdenum cofactor deficiency (MoCD) treated at Yale University School of Medicine, New Haven, CT. Born to first-cousin parents, the infant's head circumference fell from the 25 th percentile at birth to $<5$ th at age 2 months. Developmental delay, spastic quadriplegia, opisthotonus, and irritability were remarkable at 3 months, and a CT showed multiple infarcts. Focal seizures began at 4 months. Laboratory abnormalities included an elevated plasma lactate level, low plasma cysteine, low serum uric acid, and urine positive for sulfites and $S$-sulfocysteine, consistent with sulfite oxidase deficiency. The diagnosis of MoCD was confirmed by absent urinary urothione. Within one month of dietary restriction of methionine to $30 \mathrm{mg} / \mathrm{kg} / \mathrm{d}$ and supplementation of cysteine to $70 \mathrm{mg} / \mathrm{kg} / \mathrm{d}$ the infant was more active and alert, irritability resolved, head growth resumed, and seizures were controlled. Urinary sulfites were undetectable. Dietary parental noncompliance resulted in recurrence of irritability, spasticity, opisthotonus, frequent infantile spasms and hypsarrythmia, lack of head growth, regression to a vegetative state by 16 months of age, and elevated sulfites in the urine, $>400 \mathrm{mg} / \mathrm{L}$. (Boles RG et al. Short-term response to dietary therapy in molybdenum cofactor deficiency. Ann Neurol Nov 1993; $34: 742-744)$. (Respond: Dr Boles, Dep of Genetics, Yale Univ School of Medicine, WwW 305, 333 Cedar Street, New Haven, CT 06510).

COMMENT. MoCD is an autosomal recessive inborn error of metabolism involving 3 molybdenum-requiring enzymes: sulfite oxidase, xanthine dehydrogenase, and aldehyde oxidase. An infant with delayed development, microcephaly, refractory seizures, and lactic acidosis, should be tested for urinary sulfites and serum hypouricemia. Dietary therapy appears promising. The appearance of ectopia lentis, a characteristic finding, is often delayed. For MoCD as an overlooked cause of neonatal seizures, see Ped Neur Briefs July 1993; $\underline{7}: 52$.

\section{SEIZURE DISORDERS}

\section{PROGNOSIS OF INFANTILE SPASMS}

The developmental, neurologic, and seizure outcome and prognostic factors of 57 cases of infantile spasms were evaluated in relation to etiology (cryptogenic 17 cases, symptomatic 40 cases) at the Hospital for Sick Children, Toronto, Canada. Age of onset ranged from 4 weeks to 13 months (mean, 5 months), and duration of follow-up was 12 - 60 months. Cerebral dysgenesis was the most common cause (20\%) in the symptomatic group. Neurologic deficits, especially hypotonia, were present in $23 \%$ of cryptogenic cases of $75 \%$ 
of symptomatic. A mean developmental score of 71 in cryptogenic cases was significantly higher than 48 in symptomatic. Additional forms of seizures were more common in symptomatic cases (57\% cf 35\%). Unfavorable prognostic factors, particularly cognitive deficits, were the association of other seizures, neurologic deficits, delay in treatment, poor response to ACTH, and persistent EEG abnormalities after 2 weeks of therapy. All patients in the cryptogenic group responded to ACTH ( $80 \mathrm{IU} / \mathrm{IM} / \mathrm{d}$ for 2 weeks, tapered over 6 to 8 weeks). Of 30 symptomatic cases receiving ACTH, 12 (40\%) were completely controlled initially, and 11 had a partial response. Response was higher in patients with developmental scores $>70$, both cryptogenic and symptomatic cases. (Koo B et al. Infantile spasms: Outcome and prognostic factors of cryptogenic and symptomatic groups. Neurology Nov 1993; $\underline{43}: 2322-2327$ ). (Reprints: Dr Betty Koo, Division of Neurology, The Hospital for Sick Children, 555 University Avenue, Toronto, ON, Canada M5G 1X8).

COMMENT. With improved diagnostic techniques, particularly the MRI, the increased recognition of cerebral dysgenesis as a frequent cause for infantile spasms has resulted in a lowered percentage of cryptogenic cases compared to earlier reports. In a study involving 61 cases at the Mayo Clinic (Millichap JG, Bickford RG, Klass DW, Backus RE. Epilepsia 1962; 3 :188-197), 43\% were of undetermined etiology and only $7 \%$ were recognized with cerebral dysgenesis. The anticonvulsant efficacy and outcome of treatment with ACTH in 21 patients was related significantly to the age at the time of diagnosis and treatment; $80 \%$ of infants less than 1 year old were benefited whereas only $22 \%$ of those over 1 year showed reduction in seizures and EEG improvement. Control of seizures and hypsarrythmia was not significantly related to the etiology, but patients of borderline or normal intelligence tended to respond more frequently than did those with developmental quotients $<70$. (Millichap JG, Bickford RG. IAMA 1962; 182:523-527).

Posterior fossa abnormalities in children with infantile spasms were associated with a poor developmental outcome in a study of 98 children from New England Medical Center and Children's Hospital, Boston, and Hadassah University Hospital, Jerusalem (Schiffmann R et al. I Child Neurol Oct 1993; $\underline{8}: 360-365)$. Brain stem dysfunction and atrophy were remarkable in a study of 10 patients with infantile spasms, employing evoked potentials and MRI, at the University of Tokushima, Japan (Miyazaki M et al. Neuropediatr June 1993;24:126-130).

\section{BENIGN NEONATAL FAMILIAL CONVULSIONS}

An electroencephalographic-video study of 14 benign neonatal familial convulsions (BNFCs) in 3 children from two families is reported from the Hopitaux Universitaires, Strasbourg, and the Institut de Biologie, Montpellier, 